\title{
Assédio moral: evidências de validade de escala e relações com qualidade de vida no trabalho
}

\author{
Maria Isabel de Campos ${ }^{1}$, Fabián Javier Marín Rueda \\ Universidade São Francisco, Itatiba-SP, Brasil
}

\section{RESUMO}

O presente estudo teve por objetivo principal encontrar evidências de validade para a Escala Laboral de Assédio Moral - ELAM, por meio de sua correlação com a Escala de Qualidade de Vida no Trabalho - Escala-QVT. Participaram 338 trabalhadores de distintas organizações do estado de Sergipe, com idade entre 18 e 61 anos. Do total, 56,5\% (191) eram mulheres. Como resultados encontraramse correlações negativas de magnitude fraca entre os fatores das escalas, apontando evidências de validade para a ELAM. A análise de diferenças entre grupos indicou que o assédio moral laboral prejudica a percepção a respeito da qualidade de vida no trabalho.

Palavras-chave: assédio; avaliação psicológica; qualidade de vida no trabalho.

\section{ABSTRACT - Workplace bullying: Scale validity evidence and relationship to quality of work life}

The present study aimed to find validity evidence for the Workplace Bullying Scale (ELAM), through its correlation with the Quality of Work Life Scale (QVT). The participants were 338 employees of several organizations from the state of Sergipe in Brazil, aged between 18 and 61 years. Of the total, 56.5\% (191) were women. Negative correlations of low magnitude were found among the scales' factors, indicating validity evidence for ELAM. The analysis of differences between groups indicated that workplace bullying undermines the perception of the quality of work life.

Keywords: bullying; psychological assessment; quality of work life.

RESUMEN - Acoso moral: evidencias de validez para una escala y relaciones con calidad de vida en el trabajo El presente estudio tuvo como principal objetivo encontrar evidencias de validez para la Escala Laboral de Acoso Moral - ELAM, por medio de la correlación a la Escala de Calidad de Vida en el Trabajo. Participaron 338 trabajadores de diferentes organizaciones del estado de Sergipe, con edades entre 18 y 61 años. Del total, 56,5\% (191) eran mujeres. En los resultados se encontraron correlaciones negativas de baja magnitud entre los factores de las escalas, mostrando evidencia de validez para la ELAM. El análisis de diferencias entre grupos indicó que el acoso moral perjudica la percepción a respecto de la calidad de vida en el trabajo.

Palabras clave: acoso; evaluación psicológica; calidad de vida en el trabajo.

Entende-se o assédio moral no trabalho (AMT) como um conjunto de comportamentos hostis e antiéticos direcionados a um indivíduo por outro indivíduo ou grupo deles, resultando em sofrimento social e estados patológicos psicológicos ou psicossomáticos. Essas ações ocorrem de forma repetitiva (praticamente todos os dias), por um longo período de tempo - pelo menos seis meses (Leymann, 1990). O AMT passou a ocupar espaço de destaque em pesquisas da área de Psicologia a partir de estudos realizados na década de 1980 e da atenção despertada pelas publicações de Leymann $(1990 ; 1996)$. Ainda assim, alguns autores consideram o tema como sendo tão antigo quanto o próprio trabalho (Battistelli, Amazarray, \& Koller, 2011; Pamplona Filho, 2006; Ramos, 2013).
O fenômeno vem sendo estudado de forma multidisciplinar, por áreas, como Psicologia, Direito, Administração, Ciências Sociais, Medicina do trabalho e Enfermagem (Battistelli et al., 2011; Fabrício, 2012). Os estudos têm ajudado a promover a evolução do entendimento sobre o AMT, sem que se tenha atingido, no entanto, um esgotamento da necessidade de novas pesquisas.

Crawshaw (2009) sugere que as pesquisas poderiam se beneficiar da criação de uma nomenclatura padrão, que facilitaria também o desenvolvimento de melhores práticas e propostas, abrangendo interesses e envolvimento de acadêmicos, de profissionais da prática, de empregadores e de empregados. A autora baseia sua defesa na apresentação de 35 diferentes termos utilizados em inglês para nomear o fenômeno, desde abuse (abuso) até workplace 
psychological violence (violência psicológica no ambiente de trabalho) e aponta que existem conflitos de definição e de nomenclatura que prejudicam não apenas a clareza e precisão das pesquisas, mas também a definição de legislações específicas para abordagem do contexto.

Crawshaw (2009) defende, ainda, que se deve adotar uma forma descritiva para o fenômeno, evitando o excesso de definições. Afirma, também, que a perseguição no ambiente de trabalho pode envolver um ou mais perpetradores e alvos em diversos níveis da organização, ocorrer em vários graus de severidade e frequência, ser intencional ou não intencional e se manifestar por uma variedade de comportamentos inaceitáveis em resposta a vários agentes precipitantes.

No Brasil, a despeito do reconhecimento de tantas diferentes nomenclaturas para o fenômeno na língua inglesa e também em outras línguas (Pamplona Filho, 2006), essa multiplicidade de termos não parece afetar o andamento de estudos e a evolução de práticas da saúde, legislativas ou empresariais. Os múltiplos termos parecem ser bem compreendidos e aceitos como sinônimos também em Portugal, mesmo quando simplesmente adotados de outra língua (e.g. mobbing, bullying) (Guimarães \& Vasconcelos, 2012), não tendo sido encontrados trabalhos que questionem a multiplicidade de vocabulário como um obstáculo aos estudos do tema em língua portuguesa.

Ademais, Soares (2014) esclarece que a própria acepção etimológica do termo "assédio" caracteriza a repetição do ato de violência de forma insistente. Com isso, pode-se entender que os questionamentos de Crawshaw (2009) sobre a definição de Leymann (1990) não chegam a conquistar atenção ou foco das pesquisas em Psicologia, em especial no Brasil. Sob o esteio dessas considerações, neste estudo, serão utilizados diversos termos, como bullying, mobbing e outros, como sinônimos de AMT.

O AMT é considerado como um dos fatores de risco à saúde dos trabalhadores e insere-se no conjunto denominado de riscos psicossociais, comumente conhecidos como estressores organizacionais, ou características de organizações do trabalho. Pesquisas que o posicionam nesse amplo contexto têm sido desenvolvidas em diversos países europeus (Espanha, França, Itália, Portugal, Áustria, Dinamarca, Suíça, Reino Unido e Irlanda) e em outros continentes. Em conjunto com esses esforços, instituições europeias que objetivam a segurança e a saúde no trabalho têm contribuído com iniciativas voltadas à melhoria do ambiente laboral, como meio de proteger a saúde física e mental do trabalhador (UGT-CEC, 2013).

Essas medidas têm considerado o resultado de diversos estudos acadêmicos desenvolvidos desde o início deste século. Balducci, Fraccaroli e Schaufeli (2011), por exemplo, apresentaram um modelo em que fatores ambientais e de personalidade operam como antecedentes do AMT, tendo sintomas de estresse pós-traumático como resultantes do contexto. Os resultados confirmaram que exigências do ambiente laboral (carga de trabalho e conflito de papéis), bem como recursos de trabalho (autoridade de decisão, suporte de colegas e perspectivas de promoção/salário) associaram-se ao bullying e ao neuroticismo, e que o AMT mediou a relação entre a demanda de trabalho e os sintomas de estresse pós-traumático. Mais recentemente, um estudo realizado na Itália (Vignoli, Guglielmi, Balducci, \& Bonfiglioli, 2015) com a participação de 512 trabalhadores mostrou que a tensão relacionada ao trabalho é mediadora do relacionamento entre o AMT e problemas da coluna (lombar superior e inferior, e nuca).

Pesquisas nacionais também têm se preocupado em compreender o fenômeno, seus antecedentes e consequências. Em um estudo de cunho qualitativo, Torres Neto e Torres (2014) investigaram as experiências de 30 mulheres que deixaram o interior da Amazônia com o objetivo de trabalhar no Polo Industrial de Manaus, em busca de uma melhor qualidade de vida. Os autores tiveram que aplicar uma estratégia de inserção de uma pesquisadora trabalhando junto do grupo, devido à grande dificuldade de contar com a abertura dessas mulheres para relatar sua situação real. Identificaram a existência de sofrimento em vítimas de assédio e indicaram a necessidade de compreender melhor o tema para prevenir sua ocorrência, visando à melhoria da saúde e da qualidade de vida no trabalho. Como bem lembram os autores, no entanto, o método qualitativo revela uma interpretação dos fatos, que não é a única possível.

Dessa forma, é também importante compreender como têm avançado as pesquisas locais no que diz respeito ao desenvolvimento de instrumentos de medida, que venham a possibilitar a realização de uma gama de pesquisas de cunho quantitativo. Pesquisas estas que possam vir a contribuir com a criação de medidas preventivas e de intervenções efetivas na solução do problema. Nesse sentido, cabe lembrar que estudos realizados na área de Psicologia contribuem também com o desenvolvimento de diversas outras áreas do conhecimento e da prática. É assim que, em um estudo sobre a visão de operadores do direito a respeito do AMT, Battistelli et al. (2011) encontraram que, para esses operadores, a contribuição dada pelos achados de Psicologia sobre o tema relaciona-se à produção do conhecimento, à avaliação psicológica dos casos e à prevenção da violência psicológica no âmbito das organizações. As autoras concluem seu trabalho destacando a importância da integração do conhecimento multidisciplinar, com destaque especial para a atuação conjunta entre estudiosos do Direito e da Psicologia, visando à melhoria da qualidade de vida dos trabalhadores.

É de relevância, portanto, conhecer o que se tem realizado no Brasil com vistas ao desenvolvimento de novos conhecimentos em avaliação psicológica com foco em AMT. Fabrício (2012) realizou uma revisão bibliográfica sobre assédio moral em ambientes de trabalho, 
em revistas da área de Psicologia e de Administração, no período de 2010 a 2012 e apontou que das 16 publicações cujos autores são oriundos da área de conhecimento da Psicologia, apenas uma objetivava tratar de medidas de assédio. Trata-se do trabalho de Martins e Ferraz (2011), no qual as autoras relatam a adaptação e as características psicométricas de medidas de AMT com a Escala de Percepção da Frequência e a Escala de Impacto Afetivo do Assédio Moral no Trabalho, ambas resultantes da adaptação do Cuestionario de Hostigamiento Psicológico en el Trabajo (Fornés, Martínez-Abascal, \& García de la Banda, 2008).

Martins e Ferraz (2011) relatam ter localizado, na literatura internacional, apenas quatro instrumentos de medida do fenômeno, indicando, porém, não haver, no Brasil, estudos suficientes para atestar validade e fidedignidade para nenhum deles. Um dos quatro instrumentos por elas localizados é o Leymann Inventory of Psychological Terror - LIPT, validado por Leymann ainda na década de 1980 (Leymann, 1990) e que vem, desde então, sendo amplamente utilizado e passando por diversos estudos de evidências de validade, em inúmeros países. De acordo com Martins e Ferraz (2011), no entanto, tais estudos ainda não confirmaram a validade fatorial do LIPT e os índices de fidedignidade de alguns de seus fatores ainda não são considerados satisfatórios, o que teria incentivado pesquisadores a prosseguir com a construção dos outros instrumentos localizados.

Visando ampliar as medidas de AMT e estudar seu impacto sobre o estresse no trabalho, Lima e Souza (2015) desenvolveram a Escala Brasileira de Mobbing, composta por três instrumentos distintos que avaliam o AMT de acordo com os autores envolvidos, podendo ser o chefe, os colegas ou os subordinados. A escala foi desenvolvida com base no LIPT, em sua versão adaptada por González de Rivera e Rodriguez-Abuín (2005), na escala CISNEROS (Fidalgo \& Piñuel, 2004) e no NAQ - Negative Acts Questionnaire, instrumento apresentado em um congresso em Praga em 2001 e que conta desde 2009 com uma versão revisada (Einarsen, Hoel, \& Notelaers, 2009).

Estudando uma amostra de 300 servidores federais, Lima e Souza (2015) realizaram uma análise fatorial exploratória e encontraram a presença de dois fatores comportamento respeitoso e mobbing - nas três medidas (chefe, colegas e subordinados). Os autores encontraram, também, um impacto positivo significativo do mobbing sobre o estresse no trabalho. Da mesma maneira, o comportamento respeitoso por parte de chefes, colegas e subordinados teve influência negativa significativa sobre o estresse no trabalho.

Visando também contribuir com medidas de AMT, Rueda, Baptista e Cardoso (2015) desenvolveram a Escala Laboral de Assédio Moral - ELAM, com base no trabalho de Leymann (1990; 1996), que apresentava a existência de cinco estratégias adotadas nos ambientes laborais para a consecução do assédio. Para o desenvolvimento da ELAM, porém, os autores aplicaram algumas considerações sobre a dinâmica atual das organizações e decidiram dar foco a três das cinco estratégias: os limites ao contato social, o desprestígio do profissional perante seus colegas e o descrédito nas capacidades profissionais e organizacionais do profissional.

O primeiro estudo sobre a ELAM (Rueda et al., 2015) apresentou resultados que confirmaram o esperado pela literatura. Os autores, porém, discutiram a necessidade de realização de novas investigações que pudessem corroborar seus achados. Um dos tipos de investigação possível constitui o objetivo principal do estudo apresentado neste artigo: encontrar novas evidências de validade para a ELAM, por meio de sua correlação com variáveis externas.

Dada a pluralidade de medidas existentes e já validadas para o contexto dos ambientes laborais, qual delas selecionar para a concretização desse objetivo? A seleção baseou-se no entendimento da Organização Mundial de Saúde (OMS; 2003) de que o AMT "pode produzir graves consequências negativas para a qualidade de vida e na saúde dos indivíduos, principalmente nas áreas emocionais, psicossomáticas e de comportamento" (pp. 4). Baseou-se também, na convicção declarada por Soares (2014) de que, em ambientes com alta incidência de AMT, não é possível alcançar a qualidade de vida no trabalho (QVT). Assim, selecionou-se não a qualidade de vida em todos os seus domínios, como abordada pela OMS (2003), mas especificamente a que pode ser mensurada no que diz respeito exclusivamente a esse domínio, ou seja, a QVT.

A QVT pode ser compreendida como um processo dinâmico e contínuo no qual a atividade laboral organiza-se objetiva e subjetivamente, em seus aspectos operacionais e relacionais (Casas, Repullo, Lorenzo, \& Cañas, 2002). Como modelo teórico, a QVT entra no foco dos estudos de Psicologia a partir dos trabalhos de Walton (1973), que a associa tanto a aspectos de humanização do ambiente de trabalho quanto à obtenção de melhores resultados organizacionais, por meio dessa humanização e da conquista de maior bem-estar por parte dos trabalhadores. É nesse sentido que Walton (1973) relaciona, em seu modelo teórico, oito aspectos a serem analisados: a compensação justa e adequada; as condições de trabalho; o uso e desenvolvimento das capacidades dos trabalhadores; as chances de crescimento e de segurança; a integração social na empresa; o constitucionalismo; o trabalho e o contexto de vida; e a relevância social do trabalho.

Dessa forma, além de encontrar evidências de validade para as medidas resultantes da ELAM por meio de sua correlação com uma medida de QVT, é também objetivo deste estudo analisar as diferenças entre grupos de faixas distintas de pontuação de AMT, com relação à QVT e investigar se o entendimento da OMS (2003) e a conviç̧ão de Soares (2014) são respaldados por dados 
empíricos. Com o intuito de contribuir com a análise na prática organizacional, casos específicos com alta pontuação de AMT serão também investigados com relação à QVT.

As hipóteses aventadas são que as correlações existentes entre as medidas de AMT e de QVT sejam negativas e que se encontrem diferenças significativas entre grupos com distintos níveis de pontuação de AMT com relação à QVT. Espera-se que os achados deste estudo possam contribuir com o desenvolvimento do conhecimento acadêmico e também da prática organizacional, especialmente levando em conta índices levantados por pesquisas efetuadas no Brasil, tal como a realizada por Barreto (2003), que indicou que $42 \%$ dos entrevistados teriam sofrido algum tipo de AMT.

\section{Método}

\section{Participantes}

Participaram desta pesquisa 338 trabalhadores, oriundos de diferentes empresas (indústria, comércio e serviços), residentes no estado de Sergipe e com idade entre 18 e 61 anos $(M=30,19, D P=10,24)$. Dentre os pesquisados, 56,5\% (191) eram mulheres. O nível de escolaridade dividiu-se em ensino fundamental completo ou incompleto $(34,6 \%, n=117)$ e ensino médio completo ou incompleto $(65,4 \%, n=221)$. Como critérios de inclusão, foi solicitado dos respondentes que estivessem trabalhando por um período maior que um ano na mesma empresa e sob o regime de contratação pela Consolidação das Leis do Trabalho (CLT).

\section{Instrumentos}

Escala Laboral de Assédio Moral - ELAM (Rueda et al., 2015). A ELAM mede a percepção do trabalhador sobre a existência de assédio moral em seu ambiente de trabalho. Evidências iniciais de validade foram encontradas por meio de uma análise fatorial exploratória que indicou a existência de três fatores, capazes de explicar $46,45 \%$ da variância total. Composta por 27 itens distribuídos pelos três fatores, a ELAM é uma escala tipo Likert onde $1=$ nunca, $2=$ poucas vezes, $3=$ muitas vezes e $4=$ sempre. Alguns itens que exemplificam, na ordem apresentada, cada um dos três fatores da escala são: "Sou obrigado(a) a fazer tarefas que não são da minha responsabilidade", "Sofro preconceito devido a minha opção sexual" e "Sou excluído(a) das conversas em grupo".

O fator 1 (11 itens, $\alpha=0,85)$ é denominado Condições de Trabalho e se refere a dificuldades que o funcionário enfrenta em seu ambiente de trabalho, tais como tarefas fora do escopo da função, pressão e controle demasiado e indevido, desrespeito e exposição pública, entre outras. O fator 2 ( 8 itens, $\alpha=0,87$ ) é nomeado Humilhação e diz respeito a comportamentos de violência psicológica, tais como exclusão do grupo, constrangimento, aviltamento e humilhação por parte de colaboradores/chefia. O fator 3 ( 8 itens, $\alpha=0,82$ ) é denominado Preconceito e focaliza comportamentos de intolerância e discriminação por características psicológicas e/ou físicas, tais como orientação sexual, sexo, cor e raça.

Escala de Qualidade de Vida no Trabalho - EscalaQVT (Rueda, 2013). A Escala-QVT é usada para medir o grau de qualidade de vida no trabalho, sendo composta por 35 itens agregados em quatro fatores. Evidências iniciais de validade foram encontradas por meio de uma análise fatorial exploratória, cujos resultados indicaram a existência de quatro fatores, explicando uma variância total de $51,17 \%$.

Trata-se de uma escala Likert com pontuação variando desde $1=$ discordo totalmente, até $5=$ concordo plenamente. Alguns exemplos de itens são "Minha vida pessoal é respeitada", "Meu salário é justo para o esforço (físico ou mental) que realizo", "O horário de trabalho favorece o convívio social fora da empresa" e "Tenho autonomia para realizar o meu trabalho". A interpretação dos resultados é feita de acordo com a somatória da pontuação dos itens de cada fator, que podem se distribuir por cinco classificações para a QVT sendo elas baixa, média baixa, média, média alta e alta.

O fator 1 (15 itens, $\alpha=0,90)$ é denominado QVT relacionada à Integração, ao Respeito e à Autonomia e identifica a promoção do respeito pelas diferenças individuais e pelos direitos dos funcionários, a colaboração e o respeito entre os colegas e a promoção da autonomia por meio de normas e regras claras; o fator 2 (6 itens, $\alpha=0,89)$ diz respeito à Compensação Justa e Adequada e se refere à QVT relativa à satisfação com o salário e às políticas salariais da organização, percebendo que ela é justa com o funcionário nesse aspecto; o fator 3 (8 itens, $\alpha=0,82$ ) indica a QVT relacionada a Incentivo e Suporte, como treinamentos, cursos de aperfeiçoamento e incentivo à realização de atividades culturais; e o fator 4 (6 itens, $\alpha=0,84$ ) mensura a QVT relacionada a Possibilidades de Lazer e Convívio Social extra organização proporcionadas, porém, com base em características da própria organização, como carga horária, jornada e volume de trabalho.

\section{Procedimentos}

Após a pesquisa ser aprovada por um comitê de ética, iniciou-se a coleta de dados, que foi realizada sob o consentimento das empresas e em espaço cedido por elas, com agendamento prévio. Os participantes receberam as orientações necessárias à compreensão dos objetivos da pesquisa e sobre sua participação, assinaram o Termo de Consentimento Livre e Esclarecido (TCLE) e, na sequência, responderam as questões referentes a seu perfil pessoal e da empresa (sexo, idade, escolaridade, setor) e as duas escalas descritas. Os dados foram coletados em papel, posteriormente digitados em planilha eletrônica e, posteriormente, exportados para um software de análise estatística. 


\section{Análise de Dados}

Inicialmente foram calculados os escores para cada um dos fatores da ELAM e da Escala-QVT, verificados os índices de confiabilidade das escalas para a amostra por meio do alpha de Cronbach e, então, os dados foram tratados por meio de estatística descritiva. Na sequência, foi utilizada a correlação de Pearson para avaliar a existência de correlações entre os fatores da ELAM e da EscalaQVT. Por meio do teste $t$ de Student foi investigada a existência de diferenças entre os grupos extremos pontuados pela ELAM, com relação aos escores para a EscalaQVT. O tamanho do efeito - $d$ de Cohen - foi utilizado para a compreensão do impacto do AMT sobre a QVT e casos com alta pontuação de AMT foram analisados em suas especificidades.

\section{Resultados}

Os resultados obtidos para índices de confiabilidade para os fatores da ELAM indicaram $\alpha=0,86, \alpha=0,89$ e $\alpha=0,88$ para os fatores 1,2 e 3 , respectivamente. Para os fatores de 1 a 4 da Escala-QVT, os resultados indicaram $\alpha=0,94, \alpha=0,89, \alpha=0,88$ e $\alpha=0,85$, na mesma ordem. A análise estatística descritiva indicou o resultado para a média dos fatores de cada escala. Os valores são apresentados na Tabela 1.

Tabela 1

Estatísticas Descritivas para a ELAM e a Escala-QVT - $(\mathrm{N}=338)$

\begin{tabular}{|c|c|c|c|c|c|c|c|c|c|}
\hline ELAM & Mín. & Máx. & M & DP & Escala QVT & Mín. & Máx. & M & $D P$ \\
\hline Condições de trabalho & 11 & 35 & 15,73 & 5,12 & $\begin{array}{l}\text { Integração, respeito } \\
\text { e autonomia }\end{array}$ & 20 & 75 & 57,19 & 13,99 \\
\hline Humilhação & 8 & 31 & 9,77 & 3,29 & $\begin{array}{l}\text { Compensação justa } \\
\text { e adequada }\end{array}$ & 6 & 30 & 19,01 & 6,71 \\
\hline Preconceito & 8 & 27 & 9,27 & 2,91 & Incentivo e suporte & 8 & 40 & 25,03 & 8,77 \\
\hline- & & & - & - & $\begin{array}{l}\text { Possibilidades de } \\
\text { lazer e convívio social }\end{array}$ & 6 & 30 & 21,19 & 6,14 \\
\hline
\end{tabular}

Observa-se que, para os fatores da ELAM, as pontuações máximas para cada um dos fatores, 44, 32 e 32 para fatores 1, 2 e 3 respectivamente, não foram atingidas. Além disso, também para todos os fatores, as somatórias das médias e seus respectivos desvios padrão resultaram em um valor abaixo do ponto médio da pontuação máxima possível de cada fator. Para a Escala-QVT, as médias obtidas indicaram uma percepção de QVT relacionada a Integração, Respeito e Autonomia e de QVT relacionada à Possibilidades de Lazer e Convívio Social classificadas como média baixa. Para a QVT relacionada a Compensação Justa e Adequada e para a QVT relacionada a Incentivo e Suporte às médias indicaram uma percepção de QVT média (Rueda, 2013).

Após a análise descritiva dos dados, prosseguiu-se com o cálculo da correlação de Pearson. A Tabela 2 mostra os resultados encontrados.

Os resultados mostram que, das doze correlações possíveis, todas apresentaram valor negativo e dez se mostraram estatisticamente significativas. Todas as correlações encontradas apresentaram magnitude fraca (Dancey \& Reidy, 2006). Esses resultados indicam que as escalas utilizadas medem construtos diferentes e praticamente sem variância comum.

Tabela 2

Correlações entre os Fatores da ELAM e da Escala-QVT

\begin{tabular}{|c|c|c|c|c|c|c|}
\hline \multicolumn{7}{|c|}{ Escala-QVT } \\
\hline & & & $\begin{array}{c}\text { Integração, } \\
\text { respeito e } \\
\text { autonomia }\end{array}$ & $\begin{array}{l}\text { Compensação } \\
\text { justa e adequada }\end{array}$ & $\begin{array}{l}\text { Incentivo e } \\
\text { suporte }\end{array}$ & $\begin{array}{c}\text { Possibilidades de } \\
\text { lazer e convívio } \\
\text { social }\end{array}$ \\
\hline \multirow{4}{*}{ 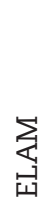 } & \multirow{2}{*}{ Condições de trabalho } & $r$ & $-0,14$ & $-0,12$ & $-0,14$ & $-0,20$ \\
\hline & & $p$ & 0,011 & 0,027 & 0,010 & 0,000 \\
\hline & \multirow{2}{*}{ Humilhação } & $r$ & $-0,16$ & $-0,14$ & $-0,09$ & $-0,16$ \\
\hline & & $p$ & 0,002 & 0,012 & 0,087 & 0,004 \\
\hline \multirow{2}{*}{\multicolumn{2}{|c|}{ Preconceito }} & $r$ & $-0,16$ & $-0,11$ & $-0,08$ & $-0,13$ \\
\hline & & $p$ & 0,004 & 0,045 & 0,141 & 0,018 \\
\hline
\end{tabular}


Visando ainda a verificar a existência de diferenças entre grupos, foram identificados e separados os grupos extremos na pontuação para todos os fatores da ELAM, para posteriormente compará-los com os fatores da Escala-QVT, por meio do teste $t$ de Student. Foram também calculados os tamanhos de efeito, usando o $d$ de Cohen. Os resultados encontram-se na Tabela 3.

Encontraram-se diferenças estatisticamente significativas entre os grupos para sete dos doze pares formados entre os fatores das escalas. Destaca-se que, para o fator Humilhação da ELAM, só não existiram diferenças significativas de pontuação entre os grupos extremos para o fator Incentivo e suporte da EscalaQVT. Para o fator Condições de Trabalho da ELAM foram encontradas diferenças estatisticamente significativas para as pontuações dos fatores Incentivo e Suporte e Possibilidades de Lazer e Convívio Social da Escala-QVT. Por fim, para o fator Preconceito da ELAM, encontraram-se diferenças significativas entre os grupos para os fatores Integração, Respeito e Autonomia, e Possibilidades de Lazer e Convívio Social da Escala-QVT.

Tabela 3

Descrição estatística dos Fatores da Escala-QVT, para os Grupos Extremos da ELAM

\begin{tabular}{|c|c|c|c|c|c|c|c|c|}
\hline \multicolumn{9}{|c|}{ Fatores Escala-QVT } \\
\hline \multicolumn{9}{|c|}{ Integração, respeito e autonomia } \\
\hline \multirow{7}{*}{ 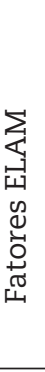 } & \multirow{3}{*}{ Condições de trabalho } & Extremo & $N$ & M & DP & $t$ & $p$ & $d$ \\
\hline & & Inferior & 80 & 57,86 & 14,23 & 1,312 & 0,192 & 0,21 \\
\hline & & Superior & 82 & 55,02 & 13,31 & & & \\
\hline & \multirow{2}{*}{ Humilhação } & Inferior & 190 & 59,09 & 13,08 & 2,406 & 0,017 & 0,29 \\
\hline & & Superior & 104 & 55,14 & 14,10 & & & \\
\hline & \multirow{2}{*}{ Preconceito } & Inferior & 222 & 58,50 & 13,44 & 2,386 & 0,018 & 0,27 \\
\hline & & Superior & 116 & 54,70 & 14,71 & & & \\
\hline \multicolumn{9}{|c|}{ Compensação justa e adequada } \\
\hline \multirow{7}{*}{ 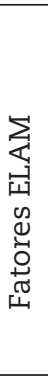 } & \multirow{3}{*}{ Condições de trabalho } & Extremo & $\mathrm{N}$ & $M$ & $\mathrm{DP}$ & $t$ & $p$ & $d$ \\
\hline & & Inferior & 80 & 19,83 & 6,64 & 1,826 & 0,070 & 0,29 \\
\hline & & Superior & 82 & 17,91 & 6,68 & & & \\
\hline & \multirow{2}{*}{ Humilhação } & Inferior & 190 & 19,98 & 6,58 & 2,487 & 0,013 & 0,30 \\
\hline & & Superior & 104 & 17,98 & 6,60 & & & \\
\hline & \multirow{2}{*}{ Preconceito } & Inferior & 222 & 19,40 & 6,58 & 1,489 & 0,137 & 0,17 \\
\hline & & Superior & 116 & 18,26 & 6,92 & & & \\
\hline \multicolumn{9}{|c|}{ Incentivo e suporte } \\
\hline \multirow{7}{*}{ 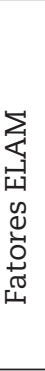 } & \multirow{3}{*}{ Condições de trabalho } & Extremo & $\mathrm{N}$ & $M$ & $\mathrm{DP}$ & $t$ & $p$ & $d$ \\
\hline & & Inferior & 80 & 26,58 & 8,95 & 2,210 & 0,029 & 0,35 \\
\hline & & Superior & 82 & 23,56 & 8,40 & & & \\
\hline & \multirow{2}{*}{ Humilhação } & Inferior & 190 & 26,07 & 8,67 & 1,541 & 0,125 & 0,19 \\
\hline & & Superior & 104 & 24,44 & 8,63 & & & \\
\hline & \multirow{2}{*}{ Preconceito } & Inferior & 222 & 25,58 & 8,74 & 1,603 & 0,110 & 0,18 \\
\hline & & Superior & 116 & 23,97 & 8,77 & & & \\
\hline \multirow{8}{*}{ 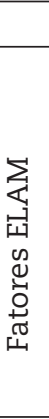 } & & Possibilic & e laze & convívi & & & & \\
\hline & \multirow{3}{*}{ Condições de trabalho } & Extremo & $\mathrm{N}$ & M & $\mathrm{DP}$ & $t$ & $p$ & $d$ \\
\hline & & Inferior & 80 & 21,78 & 6,00 & 2,467 & 0,015 & 0,39 \\
\hline & & Superior & 82 & 19,46 & 5,92 & & & \\
\hline & \multirow{2}{*}{ Humilhação } & Inferior & 190 & 22,11 & 5,88 & 2,950 & 0,003 & 0,36 \\
\hline & & Superior & 104 & 19,94 & 6,25 & & & \\
\hline & \multirow{2}{*}{ Preconceito } & Inferior & 222 & 21,67 & 6,05 & 2,004 & 0,046 & 0,23 \\
\hline & & Superior & 116 & 20,27 & 6,23 & & & \\
\hline
\end{tabular}


Por meio dos valores de $d$, de acordo com Cohen (1992), observa-se na Tabela 3 que os tamanhos de efeito mostraram-se pequenos em três dos doze pares analisados $(<0,20)$ e moderados em nove deles $(0,21 \leq d \leq 0,49)$. Isso significa que, mesmo para o caso de duas das diferenças cujos valores não indicaram significância estatística - o que pode ocorrer em função do tamanho dos grupos analisados (Cohen, 1988) - o tamanho do efeito dado pelo $d$ de Cohen mostrou-se de magnitude moderada.

Os achados apresentados na Tabela 1 indicaram não apenas que a maioria dos participantes demonstrou, por meio de suas pontuações, não se perceber como alvo de AMT, mas também que houve uma considerável dispersão dos dados. Decidiu-se, por esse motivo e visando melhor compreensão de eventuais resultados semelhantes junto a uma possível prática organizacional, analisar as pontuações dos indivíduos que marcaram os maiores escores da amostra para os fatores da ELAM. Foi identificado, na base de dados, que a frequência da pontuação máxima mostrada na Tabela 1 foi de apenas um indivíduo por fator. A Tabela 4 mostra as pontuações para esses indivíduos.

Observa-se que os indivíduos 2 e 3 pontuaram acima do ponto médio para todos os fatores da ELAM e que o indivíduo 1 só não pontuou acima do ponto médio para o fator Humilhação. Quanto à QVT, de acordo com (Rueda, 2013), os resultados indicaram que o indivíduo 1 apresentou classificação de QVT média baixa para o fator Possibilidades de Lazer e Convívio Social, classificação de QVT média para o fator Integração, Respeito e Autonomia e classificação de QVT média alta para os fatores Compensação Justa e Adequada e Incentivo e Suporte. O indivíduo 3 apresentou classificação de QVT média baixa para o fator Integração, Respeito e Autonomia e classificação de QVT baixa para os outros três fatores da escala. Por fim, observa-se que o indivíduo 2 apresentou classificação de QVT baixa para os quatro fatores.

Tabela 4

Resultados Gerais dos Indivíduos com Maior Pontuação para Cada Fator da ELAM

\begin{tabular}{|c|c|c|c|c|}
\hline & & Indivíduo 1 & Indivíduo 2 & Indivíduo 3 \\
\hline \multirow{3}{*}{ 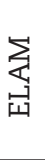 } & Condições de trabalho & 35 & 34 & 34 \\
\hline & Humilhação & 15 & 31 & 30 \\
\hline & Preconceito & 19 & 25 & 27 \\
\hline \multirow{4}{*}{ 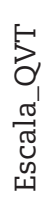 } & Integração, respeito e autonomia & 60 & 24 & 54 \\
\hline & Compensação justa e adequada & 25 & 9 & 7 \\
\hline & Incentivo e suporte & 29 & 9 & 16 \\
\hline & Possibilidades de lazer e convívio social & 20 & 10 & 13 \\
\hline
\end{tabular}

\section{Discussão}

O objetivo principal do estudo apresentado neste artigo foi encontrar evidências de validade para a ELAM, por meio de sua correlação com variáveis externas. Dada a natureza do fenômeno e os entendimentos apresentados pela OMS (2003) e por Soares (2014), a variável externa selecionada para a investigação foi a QVT, sendo esperado encontrar correlações negativas entre as medidas. Os resultados obtidos por meio da correlação de Pearson indicaram que esse objetivo foi atingido, sendo possível afirmar que foram encontradas novas evidências de validade para a ELAM.

As correlações encontradas, todas de valor negativo, indicam que quanto mais um indivíduo se percebe como alvo de AMT, menos ele percebe a existência de QVT em seu ambiente de atuação. As magnitudes das correlações encontradas foram fracas, porém, o que poderia indicar que mudanças na percepção de AMT provocariam mudanças quase inexpressivas na percepção de QVT. No entanto, parafraseando Cohen (1988), quão pequeno é um ponto? Isto é, quão inexpressivo pode ser considerado o resultado dessas mudanças?

A análise das diferenças realizada entre os grupos extremos pode ajudar a responder essa questão. Os resultados indicaram a existência de sete diferenças estatisticamente significativas entre esses grupos. Isso pode indicar que, apesar de os ambientes de trabalho amostrais não terem apresentado altos índices de AMT, contrariando, de certa forma, os achados de Barreto (2003), ainda assim, uma percepção levemente maior de atos que possam ser considerados como AMT refletem na redução da QVT e também o oposto.

As informações fornecidas pelos $d$ de Cohen possibilitam compreender o eventual impacto que variações do AMT podem causar na QVT, sejam as diferenças encontradas entre os grupos estatisticamente significativas, ou não. Tomem-se, como exemplo para análise, os resultados encontrados para os fatores Humilhação da ELAM e Integração, Respeito e Autonomia da Escala-QVT. Para 
esses fatores, foram encontradas diferenças significativas $(p=0,017)$ entre os grupos extremos, inferior e superior, e tamanho do efeito moderado $(d=0,29)$. Interpretando as informações de Cohen (1988), isso implica que, se exercida uma intervenção sobre o grupo "superior" visando à redução dos escores de Humilhação, em estudos futuros ou em uma situação prática, pode-se esperar que cerca de $60 \%$ dos participantes do grupo atinjam valores médios dos escores para integração respeito e autonomia acima da média do grupo "inferior".

Ressalta-se que a média de Integração, Respeito e Autonomia para o grupo extremo inferior indica percepção de QVT média e para o grupo extremo superior indica percepção de QVT média baixa (Rueda, 2013). Com esse entendimento, verifica-se a existência de significância prática (Conboy, 2012) para o caso. Significância essa que não pode ser considerada irrelevante, mesmo que os dados estatísticos $(r$ e $d$ ) não tenham se mostrado, em primeira instância, atraentes ou promissores.

Seguindo o mesmo raciocínio para todos os fatores combinados de ambas as escalas, verifica-se, por meio dos valores de $d$ e da normatização de resultados da Escala-QVT (Rueda, 2013), que intervenções visando à redução de AMT poderiam levar a mudanças de percepção de QVT para faixas superiores de resultados, com porcentagens de mudança de considerável significância prática. Isso ocorreria em dez das doze situações medidas, independentemente da indicação prévia de significância estatística para os dados correlacionais.

Cohen (1988) já alertava para situações desse gênero ao indicar que o uso da classificação prévia para os valores de $d$, por ele mesmo proposta, deve ser realizado apenas quando não houver uma comparação melhor. De acordo com Conboy (2012), essa afirmação de Cohen dirigia-se à busca de outros estudos similares, com os quais fosse possível contrastar os resultados obtidos, o que não é o caso deste estudo. Ellis (2010), no entanto, apresenta uma visão que resolve a questão relativa à inexistência de estudos prévios. O autor defende que, além do c de Cohen, outros dois Cs precisam ser considerados, sempre que se avaliam diferenças entre grupos. Esses Cs são o contexto e a contribuição.

Nesse sentido, o que Ellis (2010) esclarece é que a interpretação meramente estatística não é suficiente para responder a questão "quão grande é um ponto?" (Cohen, 1988, p. 21) e que interpretações de dados devem ser feitas à luz da realidade em que eles se encaixam (contexto e contribuição). O contexto em que os dados deste estudo se inserem é o ambiente organizacional, onde profissionais da prática precisam compreender diversos tipos de causas para os problemas de saúde física e mental apresentados pelos funcionários, conforme amplamente discutido na literatura (Barreto, 2003; Lima \& Souza, 2015; OMS, 2003; Soares, 2014; Torres et al., 2014; UGTCEC, 2013; Vignoli et al., 2015). Mais que isso, porém, esses profissionais precisam compreender sobre quais pontos agir/intervir e quais as relações de custo/benefício para essas ações/intervenções (contribuição).

Assim, dado que medidas do comportamento organizacional, tais como as de AMT e de QVT, podem ser utilizadas como indicadores de gestão nas organizações, as questões que um profissional da prática teria que se colocar em um contexto similar ao avaliado neste estudo poderiam ser do tipo "é realmente sobre o AMT que devemos implantar ações e intervenções visando melhorar a QVT de nossos colaboradores?"; "sobre que aspecto(s) do AMT deve-se atuar primeiro?"; "que resultados realistas (metas) podemos estabelecer como alvo?"; "que consequências esperam-se obter com esse trabalho, para os funcionários e para a organização?”.

Em busca de respostas para tais questões, a contribuição oferecida por este estudo pode ser considerada não à luz da interpretação da significância estatística, mas direcionada à significância prática (Conboy, 2012). Ao oferecer o contexto sobre o qual os dados devem ser interpretados, analisando valores de $d$ em contraste com resultados já normatizados de uma medida de QVT, torna-se possível identificar indícios de que quando o trabalho é executado sobre pressão excessiva, ou quando suas demandas são consideradas injustas (fator Condições de Trabalho da ELAM), quando as pessoas se percebem alvo de situações humilhantes e/ou alvo de preconceitos por parte de chefia e/ou de colegas (fatores Humilhação e Preconceito), elas também apresentam uma percepção menor a respeito da QVT propiciada pela existência de integração, respeito e autonomia em seu ambiente organizacional.

O mesmo é verdadeiro para percepções a respeito das possibilidades de lazer e convívio social fora do ambiente de trabalho, propiciadas pela própria organização, pois, além da possibilidade de carga horária excessiva demandada pela empresa, é possível também que ao indivíduo que sofre AMT sequer reste energia ou interesse para o lazer. Também a percepção sobre a QVT relacionada a Compensação Justa e Adequada é prejudicada, indicando que trabalhos que provocam sofrimento físico e/ ou psicológico podem não ser considerados como bem recompensados financeiramente (o dinheiro não chegaria a compensar o sofrimento).

Retoma-se, nesse ponto, que foi também objetivo do estudo investigar se dados empíricos respaldariam a afirmação da OMS (2003) de que o AMT provoca efeitos negativos sobre a qualidade de vida do trabalhador e a convicção de Soares (2014, p.45) de que "contextos de produção 'impregnados' por práticas de assédio moral no trabalho têm a qualidade de vida no trabalho (QVT) inviabilizada". Para essa análise, foram estudadas, inicialmente, as estatísticas descritivas, que mostraram que as médias da amostra ficaram abaixo da pontuação média e próximas da pontuação mínima para todos os fatores da ELAM, o que leva a entender, independentemente do fato de a ELAM ainda carecer de dados normativos, 
que a amostra não representa ambientes "impregnados" de AMT. Dessa forma, não foi possível respaldar as referenciadas afirmações quando se analisaram os resultados amostrais integrais. Para cumprir esse objetivo seriam necessários novos estudos, abrangendo ambientes em que a presença de AMT se mostre maior.

Quanto a isso, ressalta-se, porém, a dificuldade de coletar dados fidedignos sobre o tema, conforme a situação descrita por Torres et al. (2014). Uma vez que as empresas são o meio de contato entre o pesquisador e os trabalhadores, o medo de vir a sofrer eventuais consequências negativas no ambiente de trabalho pode influenciar e enviesar as respostas recebidas, reduzindo o potencial de uma medida fidedigna de AMT. O investigador deve prever essa situação e criar estratégias para contorná-la, sempre que desejar coletar dados que podem ser indesejados por empresas que, de alguma forma, promovem a existência de ambientes não salutares para os trabalhadores.

Uma vez que a amostra não possibilitou interpretar os ambientes estudados como "impregnados" de AMT (Soares, 2014, p.45), a análise de resultados voltou-se para as percepções individuais, não em busca de eventuais generalizações, mas visando mais uma vez colaborar com a interpretação prática dos dados, visto que o profissional da prática deverá agir não apenas sobre os resultados médios obtidos em sua organização, mas, principalmente, sobre casos de indivíduos que apresentem potencial de sofrimento e de prejuízo à saúde, dentro do contexto em que vivem. Dos 338 indivíduos pesquisados, apenas três apresentaram percepções de AMT com as pontuações máximas alcançadas na amostra para pelo menos um dos fatores da ELAM. Um desses três indivíduos, denominado indivíduo 1, pontuou abaixo do ponto médio para o fator Humilhação e os indivíduos 2 e 3 pontuaram acima do ponto médio em todos os fatores.

Ao pontuarem acima do ponto médio e mesmo próximos à pontuação máxima possível, esses indivíduos parecem indicar que sofrem AMT por meio das três estratégias medidas pela ELAM. Isto é, por meio da estratégia condições de trabalho, que demonstra que os indivíduos se percebem muitas vezes realizando tarefas que não são suas e que sofrem pressão ou controle excessivo e indevido (trata-se de estratégia que promove descrédito nas capacidades profissionais do indivíduo); por meio da estratégia humilhação, que abrange comportamentos de violência psicológica, tais como exclusão do grupo, constrangimento, aviltamento e humilhação por parte da chefia ou de colaboradores (trata-se da estratégia de limites ao contato social); e por meio da estratégia preconceito, que abrange comportamentos de intolerância e discriminação por características psicológicas e/ou físicas, tais como orientação sexual, sexo, cor e raça (trata-se da estratégia de desprestígio do profissional perante seus colegas).

Os indivíduos 2 e 3 indicam, também, não ter qualidade de vida no ambiente de trabalho, uma vez que ambos pontuaram QVT baixa para os fatores Compensação Justa e Adequada, Integração e Suporte, e Possibilidades de Lazer e Convívio Social. O indivíduo 3 pontuou QVT baixa também para o fator Integração, Respeito e Autonomia, enquanto que o indivíduo 2 pontuou QVT média baixa para esse fator, o que pode indicar que ele ainda se sente relativamente integrado ou um pouco respeitado dentro do grupo, ou que ainda percebe algum espaço de autonomia em seu cotidiano.

Não se pode, pela análise dos casos específicos dos indivíduos 2 e 3, dizer que os apontamentos da OMS (2003) e de Soares (2014) encontraram respaldo em dados empíricos. Pode-se, no entanto, entender que em casos específicos o AMT inviabiliza a QVT, especialmente observando-se que o AMT é medido pela percepção individual sobre ser ou não perseguido, não sendo mensurada essa percepção sobre o ambiente amplo. Ou seja, a menção a ambientes "impregnados" pelo AMT (Soares, 2014, p.45) pode ser uma eventual hipérbole, visto que o assédio se dá sobre um indivíduo e, eventualmente, sobre um grupo deles. Assim, o que esta investigação permitiu constatar foi que um indivíduo que sofre AMT indica não vivenciar QVT.

Este estudo apresenta limitações e, dentre elas, podem ser mencionadas o tamanho e as especificidades da amostra, por terem dela participado indivíduos oriundos de um único estado e com níveis de escolaridade restritos ao fundamental e médio. Outra limitação se refere ao fato de os participantes não trabalharem em uma única organização, ou não terem sido agrupados, em tempo de coleta, por organizações, o que poderia possibilitar uma análise mais ampla de ambientes de trabalho.

Independentemente dessas limitações, foi possível cumprir os objetivos de encontrar novas evidências de validade para a ELAM. Não foi possível, porém, respaldar os entendimentos da OMS (2003) e de Soares (2014) por meio de dados empíricos. Cumpriu-se, também, o objetivo de analisar as diferenças entre grupos extremos de pontuação de AMT e espera-se que essa análise, unida à análise de casos específicos com alta pontuação de AMT, possa contribuir para com questões inerentes à prática organizacional.

Novos estudos se fazem necessários, tanto no sentido de encontrar novas evidências de validade para a ELAM, aprimorando as medidas, quanto no sentido de obter informações que propiciem a sua normatização. Sugere-se, ainda, que novas pesquisas sejam realizadas de forma a mapear ambientes organizacionais distintos, tanto no que diz respeito àqueles onde se espera encontrar um alto índice de AMT, quanto àqueles onde se espera que o AMT seja ausente. Uma análise de distintos ambientes organizacionais e das percepções de seus colaboradores com relação à existência de AMT poderia, eventualmente, propiciar insights que conduzam à prevenção do fenômeno. 


\section{Referências}

Barreto, M. M. S. (2003). Violência, saúde e trabalho: uma jornada de humilhações. São Paulo: EDUC.

Balducci, C., Fraccaroli, F., \& Schaufeli, W. B. (2011). Workplace bullying and its relation with work characteristics, personality, and posttraumatic stress symptoms: An integrated model. Anxiety, Stress \& Coping, 24(5), 499-513. Recuperado de https://lirias.kuleuven.be/ bitstream/123456789/487003/1/42.pdf

Battistelli, B. M., Amazarray, M., \& Koller, S. (2011). O assédio moral no trabalho na visão de operadores do direito. Psicologia E Sociedade, 23(1), 35-45. Recuperado de http://www.scielo.br/pdf/psoc/v23n1/a05v23n1

Casas, J., Repullo, J. R., Lorenzo, S., \& Cañas, J.J. (2002). Dimensiones y medición de la calidad de vida laboral en profesionales sanitarios. Revista de Administración Sanitaria, VI(23), 143-160. Recuperado de http://rrhh-esgc.wikispaces.com/file/view/Calidad + de +vida +laboral.pdf

Cohen, J. (1992). A power primer. Psychological Bulletin, 112(1), 155-159. Recuperado de https://thehubedu-production.s3.amazonaws.com/ uploads/2522/a39eb8b3-385c-4db8-a4b4-0f5afe0c0af3/Cohen 1992 sample size.pdf.

Cohen, J. (1988). Statistical power analysis for the behavioral sciences (2n̄ ed.). Hillsdale, NJ: Lawrence Earlbaum Associates.

Conboy, J. E. (2012). Algumas medidas típicas univariadas da magnitude do efeito. Análise Psicológica, 21(2), 145-158. Recuperado de http:// publicacoes.ispa.pt/index.php/ap/article/download/29/pdf

Crawshaw, L. (2009). Workplace bullying? Mobbing? Harassment? Distraction by a thousand definitions. Consulting Psychology Journal: Practice and Research, 61(3), 263-267. Recuperado de http://psycnet.apa.org/journals/cpb/61/3/263/

Dancey, C. P., \& Reidy, J. (2006). Estatística sem matemática para psicologia usando SPSS para Windows (3 ${ }^{\mathrm{a}}$ Ed.). Porto Alegre: Artmed.

Ellis, P. D. (2010). The essential guide to effect sizes: Statistical power, meta-analysis, and the interpretation of research results. New York: Cambridge University Press.

Einarsen, S., Hoel, H., \& Notelaers, G. (2009). Measuring exposure to bullying and harassment at work: Validity, factor structure and psychometric properties of the Negative Acts Questionnaire-Revised. Work E Stress, 23(1), 24-44. doi: 10.1080/02678370902815673.

Fabrício, E. M. (2012). Revisão bibliográfica sobre assédio moral no trabalho: o estado da arte da pesquisa brasileira. Repositório Uniceub. Recuperado de http://www.repositorio.uniceub.br/bitstream/123456789/2584/3/20262155.pdf

Fidalgo, A. M., \& Piñuel, I. (2004). La escala cisneros como herramienta de valoración del mobbing. Psicothema. 16(4), 615-624. Recuperado de http://digibuo.uniovi.es/dspace/bitstream/10651/26722/1/Psicothema.2004.16.4.615-24.pdf

Fornés, J., Martínez-Abascal, M. A., \& García de la Banda, G. (2008). Análisis factorial del cuestionario de hostigamiento psicológico en el trabajo en profesionales de enfermaria. Internacional Journal of Clinical and Health Psychology, 8(1), 267-283. Recuperado de http://rrhhesgc.wikispaces.com/file/view/Calidad + de +vida + laboral.pdf

González de Rivera, J. L., \& Rodriguez-Abuín, M. J. (2005). Cuestionario de estrategias de acoso en el trabajo: El LIPT-60. Editorial EOS, Madri. Recuperado de http://www.gonzalezderivera.com/art/pdf/manual-lipt60.pdf

Guimarães, L. A. M., \& Vasconcelos, E. F. (2012). Mobbing (assédio psicológico-moral) no ambiente de trabalho: uma visão crítica contemporânea. Revista Psicologia e Saúde, 4(1), 85-93. Recuperado de http://www.gpec.ucdb.br/pssa/index.php/pssa/article/view/139

Leymann, H. (1990). Mobbing and psychological terror at workplaces. Violence and Victims, 5(2), 119-126. Recuperado de http://www. mobbingportal.com/LeymannV\%26V1990\%283\%29.pdf

Leymann, H. (1996). The content and development of mobbing at work. European Journal of Work and Organizational Psychology, 5(2), 165184. doi:10.1080/13594329608414853

Lima, T. D. F., \& Souza, M. A. D. (2015). O impacto do mobbing sobre o estresse no trabalho. Estudos e Pesquisas em Psicologia, 15 (2), 608-630. Recuperado de http://pepsic.bvsalud.org/scielo.php?pid=S1808-42812015000200010\&script=sci_arttext

Martins, M. D. C. F., \& Ferraz, A. M. S. (2011). Propriedades psicométricas das escalas de assédio moral no trabalho: percepção e impacto. Psico USF, 16(2), 163-173. doi: 10.1590/S1413-82712011000200005

Organização Mundial da Saúde (OMS). (2003). Raising awareness of psychological harassment at work. Protecting Workers' Health Series, 4. Genebra. Recuperado de http://www.who.int/occupational_health/publications/en/oehharassmentc.pdf

Pamplona Filho, R. (2006). Noções conceituais sobre o assédio moral na relação de emprego. Jus Navigandi, 11, 347-360. Recuperado de http://coad.com.br/app/webroot/files/trab/pdf/ct net/2006/ct3806.pdf

Ramos, A. C. P. P. (2013) Assédio Moral no ambiente Taboral. Âmbito Jurídico, XVI(112). Recuperado de http://www.ambito-juridico.com.br/ site/index.php/?n_link =revista_artigos_leitura\&artigo_id $=13359$ \&revista_caderno $=25$

Rueda, F. J. M., Baptista, M. N., \& Cardoso, H. F. (2015). Construção e estudos psicométricos iniciais da escala laboral de assédio moral (ELAM). Avaliação Psicológica, 14(1), 57-68. Recuperado de http://pepsic.bvsalud.org/scielo.php?pid=S1677-04712015000100005\&script=sci_arttext

Rueda, F. J. M. (2013). Escala de avaliação de qualidade de vida no trabalho (Escala-QVT). São Paulo: Casa do Psicólogo.

Soares, L. Q. (2014). Combate ao assédio moral no trabalho: uma das preocupações centrais para a viabilização da qualidade de vida no trabalho. In Anais do XIV Congresso de Stress da ISMA-BR e XVI Fórum Internacional de Qualidade de Vida no Trabalho, (pp. 45-66). Recuperado de http://www.sinal.org.br/and/2014/wp-content/uploads/2014/09/2ServPLeandro_Queiroz_Soares-1.pdf

Torres Neto, D. G., \& Torres, L. D. O. M. (2014). El sufrimiento en el trabajo e el acoso moral sobre mujeres que laboran en el polo industrial de Manaus - AM. American Research Thoughts, 1(1), 403-421. Recuperado de http://researchthoughts.us/UploadedArticle/36.pdf

Unión General de Trabajadores - Comisión Ejecutiva Confederal [UGT-CEC]. (2013). International Year Book on Psychosocial Risks Prevention and Quality of Life at Work. Evaluation Development of Psychosocial Risks in Europe. Espanha. Recuperado de http://www.lip.univ-savoie.fr/ uploads/PDF/1570.pdf

Vignoli, M., Guglielmi, D., Balducci, C., \& Bonfiglioli, R. (2015). workplace bullying as a risk factor for musculoskeletal disorders: The mediating role of Job-Related psychological strain. BioMed Research International, 2015, 1-8. doi: 10.1155/2015/712642.

Walton. R. E. (1973). Quality of working life: what is this? Sloan Management Review, 15(1), 11-21.

recebido em maio de 2015 reformulado em novembro de 2015 aprovado em novembro de 2015

\section{Sobre os autores}

Maria Isabel de Campos é doutoranda e Mestra em Psicologia pela Universidade São Francisco. Especialista em Psicologia Transpessoal e em Administração Empresarial, e Bacharel em Ciência da Computação.

Fabián Javier Marín Rueda é Psicólogo, Mestre e Doutor em Psicologia pela Universidade São Francisco. Docente da graduação e do Programa de Pós-graduação stricto sensu em Psicologia da Universidade São Francisco. Bolsista Produtividade do CNPq. 